\title{
A new needle on the block: EchoTip ProCore endobronchial ultrasound needle
}

This article was published in the following Dove Press journal:

Medical Devices: Evidence and Research

29 March 2016

Number of times this article has been viewed

\section{H Erhan Dincer' \\ Rafael Andrade ${ }^{2}$ \\ Felix Zamora' \\ Eitan Podgaetz ${ }^{2}$}

'Division of Pulmonary, Allergy, Critical Care and Sleep Medicine, ${ }^{2} \mathrm{Section}$ of Thoracic and Foregut Surgery, University of Minnesota, Minneapolis, MN, USA
Correspondence: $\mathrm{H}$ Erhan Dincer Division of Pulmonary, Allergy, Critical Care and Sleep Medicine, 420 Delaware St SE, MMC 276, Minneapolis, MN 55455, USA

Tel + I 6126240999

Fax +l 612625 2174

Email erhan_dincer@yahoo.com
Abstract: Endobronchial ultrasound has become the first choice standard of care procedure to diagnose benign or malignant lesions involving mediastinum and lung parenchyma adjacent to the airways owing to its characteristics of being real-time and minimally invasive. Although the incidence of lung cancer has been decreasing, it is and will be the leading cause of cancerrelated mortality in the next few decades. When compared to other cancers, lung cancer kills more females than breast and colon cancers combined and more males than colon and prostate cancers combined. The type of lung cancer has changed in recent decades and adenocarcinoma has become the most frequent cell type. Prognosis of lung cancer depends upon the cell type and the staging at the time of diagnosis. The cell type and molecular characteristics of adenocarcinoma may allow individualized targeted treatment. Other malignant conditions in the mediastinum and lung (eg, metastatic lung cancers and lymphoma) can be biopsied using endobronchial ultrasound needles. Endobronchial ultrasound needle biopsies provides mostly cytology specimens due to its small sizes of needles ( 22 gauge or larger) which may not give enough tissue to make a definitive diagnosis in malignant (eg, lymphoma) or benign conditions (eg, sarcoidosis). EchoTip ProCore endobronchial needle released in early 2014 provides histologic biopsy material. Larger tissue biopsies may potentially provide a higher diagnostic yield and it eliminates mediastinoscopy or other surgical interventions. Here we aim to review bronchoscopic approach in the diagnosis of mediastinal lesions with emphasis of EchoTip ProCore needles.

Keywords: endobronchial ultrasound, lymph node biopsy, EchoTip ProCore, lung cancer, transbronchial needle aspiration

\section{Current endobronchial ultrasound technology and needles}

The convex probe endobronchial ultrasound bronchoscope (CP-EBUS by Olympus, Center Valley, PA, USA), with its convex ultrasound transducer with a frequency of $7.5 \mathrm{MHz}$ located at the tip, generates a $9 \mathrm{~cm}$ deep ultrasound image in addition to endoscopic image. The ultrasound probe is located perpendicularly and covers 50 degrees of image when it touches the airway mucosa itself or via an inflated balloon with saline. While the ultrasound probe provides real-time images of mediastinal and hilar structures, the endoscopic image allows to examine the airways with an obliquely angled forward view at 35 degrees. The CP-EBUS bronchoscopes have a $6.7 \mathrm{~mm}$ outer diameter and $6.9 \mathrm{~mm}$ at the tip. The size of the bronchoscope and the oblique endoscopic view may make airway examination and tracheal intubation difficult and require larger endotracheal tube $(8 \mathrm{~mm}$ or larger) when done in mechanically ventilated patients. 
A hybrid EBUS bronchoscope (H-EBUS, Fujifilm, Valhalla, NY, USA) has recently been marketed. H-EBUS has characteristics of a smaller outer diameter of $6.7 \mathrm{~mm}$, only 10-degree of oblique view and 130-degree of flexion. ${ }^{1}$ Not surprisingly, H-EBUS bronchoscope revealed a better airway examination to the level of segmental bronchus as compared to CP-EBUS and revealed potential to obviate the need of a conventional bronchoscope. ${ }^{2}$ A prototype of a thinner EBUS (BF-Y0046, Olympus, Olympus America, Inc., Center Valley, PA, USA) bronchoscope (outer diameter of $5.9 \mathrm{~mm}$ ) has been introduced aiming to reach more distal into the bronchial tree, including the ability to access upper lobes, and an improved flexion angle of 170 degrees. ${ }^{3}$

Currently, there are three different needle types manufactured by device companies.

ViziShot aspiration needles (Olympus America, Inc., Center Valley, PA, USA) are available in two different sizes; 21 gauge (NA-201SX-4021) and 22 gauge (NA-201SX-4022). These needles provide cytology samples and are designed to be used for Olympus EBUS bronchoscopes.

SonoTip EBUS Pro and Pro Flex needles (Medi Globe, Achenmuhle, Germany) feature the needle comprising nitinol to avoid a permanent bend or kink. The size of needles is 22 gauge with length outside the plastic sheath of $0-4 \mathrm{~cm}$, similar to ViziShot needles. SonoTip needles are compatible with Olympus, Fujifilm, and Pentax EBUS (Pentax Medical, Montvale, New Jersey, USA) bronchoscopes.

EchoTip ProCore HD (High Definition, Cook Endoscopy Inc., Limerick, Ireland) needles are available in different sizes including 22 and 25 gauge with an adjustable needle extension up to $5 \mathrm{~cm}$. These needles are compatible with Olympus and Pentax EBUS bronchoscopes and designed with core trap close to the tip of the needle that receives the tissue sample while getting fine-needle aspiration (FNA) through the needle tip (Figure 1). This is thought to collect core biopsy for histologic evaluation as opposed to only cytological material with other needles. Moreover, 25 gauge EctoTip ProCore needle is claimed to be $33 \%$ more flexible by the manufacturer than a conventional 22 gauge needle that will allow more accessibility within the lung or mediastinum.

\section{When to use endobronchial ultrasound}

Endobronchial ultrasound-transbronchial needle aspiration (EBUS-TBNA) is indicated to sample the mediastinal, hilar lymph nodes and masses, as well as the centrally located lesions adjacent to the airways (Tables 1 and 2). ${ }^{4}$

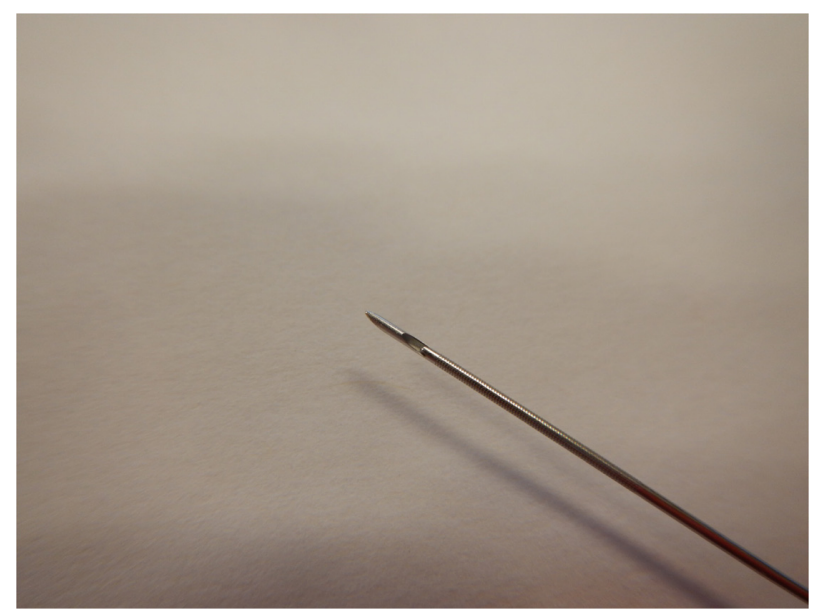

Figure I EchoTip ProCore needle, 25 gauge, showing needle tip and the reversebeveled site.

EBUS sampling of mediastinal lymph nodes has become standard of care for the staging of non-small cell lung cancer (NSCLC). In select patients NSCLC, accurate restaging of the mediastinum after induction therapy is crucial in determining prognosis and subsequent treatment. Imaging studies, such as computed tomography (CT) and positron emission tomography (PET) scans; require tissue sampling to confirm the accuracy of mediastinal restaging. As in the initial staging of the mediastinum, minimally invasive endosonographyguided needle sampling techniques such as endobronchial ultrasound-guided fine-needle aspiration (EBUS-TBNA) and endoscopic ultrasound-guided fine-needle aspiration

Table I Mediastinal lymph node stations (IASLC Lymph Node Map 2009)

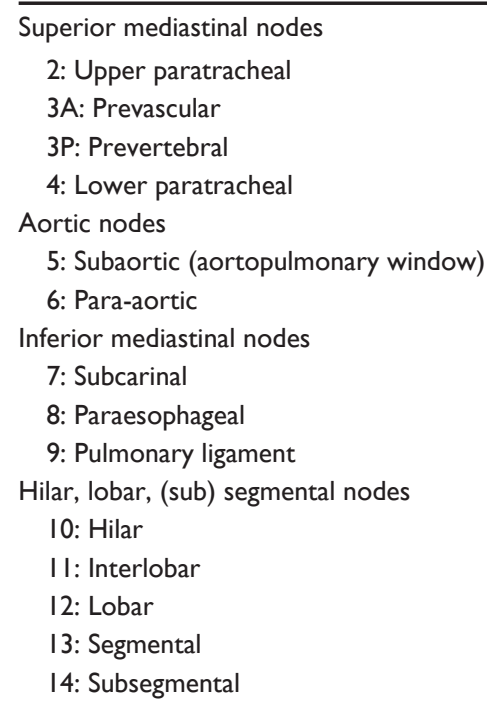

Notes: NI nodes: 10, II, 12, 13, 14; N2 nodes: 2, 4, 5, 6, 7, 8, 9; N3 nodes: contralateral mediastinal or contralateral hilar nodes or any scalene or supraclavicular nodes (station I).

Abbreviation: IASLC, The International Association for the Study of Lung Cancer, 
Table 2 Nodal station accessibility by procedures

EBUS-TBNA: 2R, 2L, 4R, 4L, 7, IOR, IOL, II, I2

EUS-FNA: 4L, 5, 6, 7, 8, 9

Mediastinoscopy: 2R, 2L, 4R, 4L, 7

Chamberlain: 5, 6

Abbreviations: EBUS, endobronchial ultrasound; EUS, endoscopic ultrasound; FNA, fine-needle aspiration; TBNA, transbronchial needle aspiration.

(EUS-FNA) are reliable in restaging the mediastinum, though surgical restaging procedures remain the gold standard. Despite a lower sensitivity in the mediastinal restaging of NSCLC, EBUS-TBNA combined with or without EUS-FNA are the preferred initial mediastinal restaging technique. Mediastinal lymphadenopathy due to benign conditions such as tuberculosis or sarcoidosis can also be readily diagnosed by EBUS-TBNA. EBUS-TBNA can access and sample lymph node stations 2 (upper paratracheal), 4 (lower paratracheal), 7 (subcarinal), 10 (hilar), 11 (interlobar), 12 (lobar) through the tracheobronchial tree, and stations 8 (paraesophageal) and 9 (pulmonary ligament) through the esophagus $(1,2)$. While stations 5 and 6 are not reachable by EBUS, EUS can have access to station 5 and station 6 (transaortic).

\section{Lung cancer staging and restaging}

TNM classification of NSCLC determines the prognosis and directs the therapeutic options. Mediastinal staging is the necessary step to identify the involvement of mediastinum. There are noninvasive and invasive methods to stage the mediastinum, however, the least invasive method that can make the diagnosis and staging in same setting is preferred. ${ }^{5}$

Noninvasive methods include imaging studies. Among those, CT scan has limited value by itself and can only stage $50 \%$ of the cancer patients, with $25 \%$ of over staged (false positive) and remainder under staged (false negative). ${ }^{6,7}$ On the other hand, PET scan is more accurate than CT scan in detecting mediastinal involvement with the advantage of showing extrapulmonary metastasis. PET scan in detecting NSCLC has a sensitivity of $84 \%$, with an excellent negative predictive value of 0.93 and specificity of $89 \%$ and a positive predictive value of $0.79 .{ }^{8-10}$

When PET and CT scans are combined (PET/CT fusion study) the diagnostic yield is better than either of them used alone in detecting the tumor size and location, nodal involvement, and distant metastasis. ${ }^{11,12}$

Drawback of PET scanning is false positivity that requires histologic verification for PET avid lesions in appropriate clinical setting. Therefore, the old adage "tissue is the issue" is still valid. Methods to obtain tissue samples include: conventional bronchoscopy with TBNA, EBUS-TBNA,
EUS-FNA, CT-guided transthoracic needle aspiration, mediastinoscopy, and video-assisted thoracoscopic surgery. While transthoracic needle aspiration has no value when sampling the mediastinum, it can be the first choice of diagnostic procedure due to its very high yield approaching to $90 \%-95 \%$ for peripheral lung lesions without mediastinal lymph node enlargement in benign or malignant conditions. ${ }^{13}$

EBUS-TBNA is superior to conventional TBNA due to its real-time imaging capability when diagnosing mediastinal lesions other than station 7. EBUS-TBNA is also found to be superior to CT and PET scanning with sensitivity (92.3\% vs $76.9 \%$ and $80 \%$, respectively), specificity (100\% vs $55 \%$ and $70 \%$, respectively), and accuracy ( $98 \%$ vs $61 \%$ and $73 \%$, respectively) in lung cancer patients. ${ }^{14}$

Restaging mediastinum after induction treatment is important to determine further treatment options. Although mediastinocopy is considered gold standard for mediastinal restaging, less invasive methods such as EBUS, EUS, or combination can be the initial method. ${ }^{15}$

\section{Diagnostic value of EBUS sampling in benign disease and lymphoma}

EBUS-TBNA is demonstrated to be an excellent diagnostic procedure in benign diseases such as sarcoidosis and tuberculosis. Recent studies have found diagnostic yield of EBUS-TBNA as 78\% in sarcoidosis. ${ }^{16}$ Other studies even reported higher sensitivity $(85 \%-93 \%)$ in patients with lymph node $>1 \mathrm{~cm}$ in diameter and if a CT scan is available before EBUS-TBNA sampling. ${ }^{17,18}$

Diagnosis of lymphoma, especially in certain types, requires larger biopsies and specific handling. Presence of on-site cytological evaluation may be helpful and prompt bronchoscopist to obtain more tissue when there is suspicion of lymphoproliferative disease. Core biopsy, in this situation, can be very useful to provide more tissue with less number of biopsy passes with the potential benefit of less procedure time, complication, and sedation use. Diagnostic yield of EBUSTBNA in lymphoma has been reported as $60 \%-90 \%{ }^{19,20}$

\section{What are the limitations and contraindications of EBUS?}

Limitations of EBUS are threefold: 1) Technical difficulties such as thorough airway examination due to oblique view, inability to access some lymph node stations (no access to stations 5, 6, and difficulty to access stations 8 and 9 via esophagus); 2) operator-related requirements such as time and training; and 3) false negative or false positive results requiring surgical intervention for confirmation. 
Contraindications of EBUS are not different than those for flexible bronchoscopy including life-threatening arrhythmias, decompensated heart failure, compromised upper airway, and uncooperative or unwilling patient. ${ }^{21}$

Bleeding risk due to antiplatelet or anticoagulation treatments, severe thrombocytopenia, and elevated blood urea nitrogen and creatinine levels can also be prohibitive for EBUS sampling. While needle lung or lymph node biopsies are considered safe on aspirin, it is recommended to hold Coumadin (3-4 days), unfractionated heparin (4-6 hours), low-molecular-weight heparin (12 hours), and antithrombin treatment ( 2 days).

It is also recommended to postpone EBUS after a myocardial infarction (at least 6 weeks), in the presence of ongoing myocardial ischemia, arrhythmia, or severe hypoxemia at rest.

\section{Benign versus malignant ultrasonographic characteristics of lymph nodes}

Several ultrasonographic findings of mediastinal lymph nodes have reported to be suggesting malignancy. Definition is an abnormal mediastinal lymph node is either short axis larger than $1 \mathrm{~cm}$ or PET positivity. ${ }^{12}$ However, both smaller lymph nodes $(<1 \mathrm{~cm})$ and nodes without PET activity can be positive for malignancy. ${ }^{22,23}$

Therefore, it is the common practice to sample lymph nodes measuring $5-10 \mathrm{~mm}$ in situations where clinical suspicion of cancer is high.

Besides the diameter, other proposed EBUS characteristics of lymph nodes suggesting malignancy are distinct round margins, heterogeneous and hyperechogenity, coagulation necrosis, and absence of central hilar structures. ${ }^{24}$

\section{Value of rapid on-site evaluation on endobronchial ultrasound}

Rapid on-site evaluation (ROSE) offers several advantages and allows bronchoscopist to make or change plan during the procedure on the basis of the immediate findings.

Although ROSE has not provided an increase in diagnostic yield in EBUS-TBNA procedures, it was found to be cost-effective, shorten the procedure time, lessen the sedation medication use and potential to decrease the complication rates.

It also allows physicians to obtain more tissue for molecular studies or flow cytometry if the initial evaluation is suspicious for adenocarcinoma or lymphoma..$^{25-27}$

\section{How to handle EBUS-TBNA specimens}

In the era of individualized and targeted treatment of lung cancer (eg, adenocarcinoma), EBUS-TBNA sampling is the least invasive way to obtain enough tissue for a definitive diagnosis, and further molecular studies such as gene alterations and mutations are conducted to determine whether the tyrosine kinase inhibitors are an option or not. ${ }^{28,29}$

There are some variations in tissue handling among different institutions. Cytological specimens are usually prepared on air-dried slides or slides fixed in 95\% alcohol for Giemsa, Diff-Quick, or Papanicolaou staining. Further testing are as follows; sample is collected in saline for culture, in 10\% formalin for cell block, DNA/RNA analysis, immunohistochemistry and fluorescence in situ hybridization, and in Roswell Park Memorial Institute medium for flow cytometry. ${ }^{30}$

\section{Role of aspiration during EBUS- TBNA}

EBUS-TBNA needles are designed with stylet that blocks the hollow of the needle to collect bronchial mucosa and wall structures during needle insertion into a lymph node or a mass. After the needle is inserted into the target lesion, the stylet is usually withdrawn and suction is applied while the needle moved back and forth in the lesion. A prospective randomized trial found no difference in sample adequacy and diagnostic yield when compared to suction and no suction during TBNA sampling of 192 lymph nodes. ${ }^{31}$

Although there is no obvious benefit of suctioning it is customary to use suction unless the lesion is vascular. In our practice, we do not use suction if the first aspiration appears to be bloody. One potential problem in suctioning vascular lesions is diluting the specimen.

\section{Needle types, sizes, and number of passes}

Currently, there are three commercially available EBUS needles: ViziShot (Olympus), SonoTip (Medi Globe), and EchoTip ProCore needles (Cook Medical, Cook Medical Inc., Bloomington, IN, USA). EchoTip ProCore designed to provide core biopsy for histologic sampling compared to others with sizes of 21 or 22 gauge, obtains only cytologic specimens. Cytology versus histology is important when diagnosing diseases that require higher cell count or larger tissue such as certain types of lymphoma (Hodgkin's disease, mantle cell, Burkitt's or large cell), adenocarcinoma 
or sarcoidosis. There are no prospective comparative studies between EchoTip ProCore versus other needles in diagnosis of pulmonary diseases. One prospective study is, however, underway, which compares 22 gauge ProCore needle with a standard 22 gauge needle. ${ }^{32}$

Desai et $\mathrm{a}^{33}$ used 22 gauge EchoTip ProCore needle in 17 patients to sample station 7 (50\% of patients), station 11 (33\%), and station 12 (17\%). Diagnosis was achieved in 89\% of patients; sarcoidosis in 12/16 patients and malignancy in $4 / 16$ patients.

This is similar to EBUS sensitivity and accuracy, 91\% and $92 \%$, respectively. ${ }^{34}$ In another retrospective study, Zambon et $\mathrm{l}^{35}$ utilized either 22 or 25 gauge ProCore needle if ROSE did not have diagnostic material after first lymph node biopsy with FNA (22 gauge conventional needle). Definitive diagnosis was achieved in 16 of 19 patients from EchoTip ProCore needle and 15 of 19 patients from conventional needle. Diagnostic yield was the highest when both needles are used, 18 of 19 patients, that is probably due to larger amount of tissue obtained.

A retrospective study compared ViziShot 22 gauge needle with SonoTip Pro and found higher diagnostic yield with SonoTip needle (74\% vs 61\%, $P=0.0035) .{ }^{36}$

A 22 gauge EchoTip ProCore needle is directly compared with a standard 22 gauge needle during endoscopic ultrasound with similar cellblock material, adequacy, accuracy of diagnosis, and complication rates. There was, however, a statistically significant trend toward fewer passes with the ProCore needle. ${ }^{37}$

The number of passes required for an optimal diagnostic accuracy has been studied. Lee et $\mathrm{al}^{38}$ reported a $100 \%$ diagnostic accuracy at third pass, without ROSE. They noted no further increase in sensitivity on further biopsy passes and concluded that three passes were enough. In another study, Trisolini et $\mathrm{al}^{39}$ showed availability of ROSE could decrease the number of biopsies needed to establish a diagnosis.

A 22 gauge needle is typically used for EBUS-TBNA sampling. A prospective study comparing 22 gauge needle to 21 gauge needle revealed no change in diagnostic yield in cancer although number of cancer cells and more preserved histologic specimens were obtained with 21 gauge needle. ${ }^{40}$

\section{Differences between EBUS needles}

Although there are no prospective comparative studies showing superiority of one needle to another, each needle was designed to improve diagnostic yield. SonoTip EBUS needles comprising nitinol (nickel and titanium alloy) that creates resistance to permanent bends and kinks when the needle advanced to curved or angulated positions. This also helps the needle to stay straight for repeated passes. SonoTip needles have also a design of twist-lock technology for precise sheath and needle length adjustments. Most experienced bronchoscopists do not necessarily use the length adjustment, however, it might be imperative for new learners. When a ViziShot needle is used in multiple samplings or in a hard, fibrous lymph nodes, it potentially can bend or kink. Nitinol comprised needle may have theoretical advantage in those situations. EchoTip ProCore needles designed to collect cytology and histology (core biopsy) specimens at the needle tip as well as the reverse-beveled side of the needle, respectively. The needle surface is dimpled to create high definition ultrasound image. Core tissue samples with 22 and 25 gauge EchoTip needles will probably allow practitioners to fewer passes and there is also less need to consider surgical sampling.

\section{Mediastinal lymph node sampling with miniforceps and needle forceps} Despite larger sizes of EBUS needles and their design to provide core tissue, the amount of tissue that recovered from needle aspirates can be limited or found insufficient by the pathologist. Miniforceps combining EBUS has been utilized in order to obtain larger biopsy specimens. ${ }^{41,42}$

Chrissian et $\mathrm{al}^{43}$ demonstrated safety and effectiveness of EBUS-guided miniforceps (1 mm miniforceps) biopsies for sampling of mediastinal and hilar lymph node enlargements. In this prospective study, the diagnostic yield of EBUS-TBNA and EBUS-guided miniforceps were $81 \%$ and $91 \%$, respectively, in patients with low likelihood of NSCLC. Neither complication rates nor procedure duration was significantly different.

More recently, EBUS-guided needle forceps were introduced. Similar to EBUS needles, the needle forceps is protected by a plastic sheath (external diameter, $1.5 \mathrm{~mm}$; length, 1,020 nm) while it is advanced through the working channel. After the needle punctured through the bronchial wall into the lymph node, the forceps with serrated jaws is opened in the lymph node to obtain tissue. In a pilot study, the histologic diagnosis was achieved in $86 \%$ of the patients with benign or malignant conditions. ${ }^{44}$

Similarly, Darwiche et $\mathrm{al}^{45}$ sampled mediastinal and hilar lymph nodes with EBUS-guided needle forceps. They compared needle forceps biopsies to EBUS-TBNA and the combination of both techniques. EBUS-guided needle 
forceps sampling increased the diagnostic yield of EBUSTBNA from $64 \%$ to $93 \%$ in benign conditions.

\section{Conclusion}

EBUS-guided TBNA is now the preferred first method to sample enlarged mediastinal or hilar lymph nodes in benign and malignant conditions. While cytological needle sampling provides high diagnostic yield, the tissue obtained with needle aspirations may not be sufficient. In order to overcome the potential low diagnostic yield of small amount of tissue, new needles and needle forceps have been developed. Although there are no prospective comparative studies, the diagnostic efficiency of the new needles has some theoretical advantages. Among these needles, SonoTip EBUS ProFlex needles are made of nitinol that has potential to avoid bending or kinking when doing multiple passes. EchoTip ProCore needle has a potential advantage in obtaining histology specimen with its core trap characteristic and is 33\% more flexibile than ViziShot. Needle forceps are designed to access easily into a lymph node when closed (needle) and to obtain larger biopsies when open (forceps). Efficacy and comparison of these needles and needle forceps are yet to be shown.

\section{Disclosure}

The authors report no conflicts of interest in this work.

\section{References}

1. Xiang Y, Zhang F, Akulian J, Yarmus L, Feller-Kopman D, Wang KP. EBUS-TBNA by a new Fuji EBUS scope (with video). J Thorac Dis. 2013;5:36-39.

2. Yarmus L, Akulian J, Ortiz R, et al. A randomized controlled trial evaluating airway inspection effectiveness during endobronchial ultrasound bronchoscopy. J Thorac Dis. 2015;7(10):1825-1832.

3. Wada HH, Hirohashi K, Nakajima T, et al. Assessment of the new thin convex probe endobronchial ultrasound scope: a preliminary study in the porcine lung. Chest. 2013;144:824A.

4. Dincer HE, Podgaetz E, Andrade R. Endoscopic ultrasound and/or endobronchial ultrasound guided needle biopsy of central intraparenchymal lung lesions not adjacent to airways or esophagus. Endosc Ultrasound. 2015;4(1):40-43.

5. Dillemans B, Deneffe G, Verschakelen J, Decramer M. Value of computed tomography and mediastinoscopy in preoperative evaluation of mediastinal nodes in non-small cell lung cancer. A study of 569 patients. Eur J Cardiothorac Surg. 1994;8:37-42.

6. Bonomo L, Ciccotosto C, Guidotti A, Storto ML. Lung cancer staging: the role of computed tomography and magnetic resonance imaging. Eur J Radiol. 1996;23:35-45.

7. Prenzel KL, Mönig SP, Sinning JM, et al. Lymph node size and metastatic infiltration in non-small cell lung cancer. Chest. 2003;123: 463-467.

8. Toloza EM, Harpole L, McCrory DC. Noninvasive staging of nonsmall cell lung cancer: a review of the current evidence. Chest. 2003; $123: 137 \mathrm{~s}-146 \mathrm{~s}$

9. Yasufuku K, Nakajima T, Motoori K, et al. Comparison of endobronchial ultrasound, positron emission tomography, and CT for lymph node staging of lung cancer. Chest. 2006;130:710-718.
10. Silvestri GA, Gould MK, Margolis ML, et al; American College of Chest Physicians. Non invasive staging of non-small cell lung cancer: ACCP evidenced based clinical practice guidelines (2nd edition). Chest. 2007;132:178S-201S.

11. Antoch G, Stattaus J, Nemat AT, et al. Non-small lung cancer: dual modality PET/CT in preoperative staging. Radiology. 2003;229:526-533.

12. De Wever W, Ceyssens S, Mortelmans L, et al. Additional value of PET$\mathrm{CT}$ in the staging of lung cancer: comparison with $\mathrm{CT}$ alone, PET alone and visual correlation of PET and CT. Eur Radiol. 2007;17:23-32.

13. Hiraki T, Mimura H, Gobara H, et al. CT fluoroscopy guided biopsy of 1000 pulmonary lesions performed with 20 -gauge coaxial cutting needles: diagnostic yield and risk factors for diagnostic failure. Chest. 2009;136:1612-1617.

14. Herth F, Becker HD, Ernst A. Conventional vs endobronchial ultrasoundguided transbronchial needle aspiration: a randomized trial. Chest. 2004; 125:322-325.

15. Khoo KL. Mediastinal re-staging of non small cell lung cancer. Thorac Cancer. 2012;3(2):145-149.

16. Medford AR, Agrawal S, Bennett JA. Sarcoidosis: technique to enable diagnosis. BMJ. 2009;339:766-767.

17. Garwood S, Judson MA, Silvestri G, Hoda R, Fraig M, Doelken P. Endobronchial ultrasound for the diagnosis of pulmonary sarcoidosis. Chest. 2007;132:1298-1304.

18. Wong M, Yasufuku K, Nakajima T, et al. Endobronchial ultrasound: new insight for the diagnosis of sarcoidosis. Eur Respir J. 2007;29:1182-1186.

19. Kennedy MP, Jimenez CA, Bruzzi JF, et al. Endobronchial ultrasoundguided transbronchial needle aspiration in the diagnosis of lymphoma. Thorax. 2008;63:360-365.

20. Steinfort DP, Conron M, Tsui A, et al. Endobronchial ultrasound-guided transbronchial needle aspiration for the evaluation of suspected lymphoma. J Thorac Oncol. 2010;5:804-809.

21. Du Rand IA, Blaikley J, Booton R, et al; The British Thoracic Society Bronchoscopy Guideline Committee: a sub-Committee of the Standards of Care Committee of the British Thoracic Society. British Thoracic Society guidelines on diagnostic flexible bronchoscopy. Thorax. 2001;56(Suppl 1):11-21.

22. Herth FJ, Ernst A, Eberhardt R, Vilmann P, Dienemann H, Krasnik M. Endobronchial ultrasound-guided transbronchial needle aspiration of lymph nodes in the radiologically normal mediastinum. Eur Respir $J$. 2006;28:910-914.

23. Gonzalez-Stawinski GV, Lemaire A, Merchant F, et al. A comparative analysis of positron emission tomography and mediastinoscopy in staging non-small cell lung cancer. $J$ Thorac Cardiovasc Surg. 2003;126:1900-1905.

24. Memoli JS, El-Bayoumi E, Pastis NJ, et al. Using endobronchial ultrasound features to predict lymph node metastasis in patients with lung cancer. Chest. 2011;140(6):1550-1556.

25. Layfield LJ, Bentz JS, Gopez EV. Immediate on-site interpretation of fine-needle aspiration smears: a cost and compensation analysis. Cancer. 2001;93:319-322.

26. Boyan LCW. On-site cytopathological analysis of bronchoscopic needle aspiration. J Bronchol. 2003;10:152-154.

27. Labib MM, Qureiyeh F, Zias N, Lamb CR. Comparison of conventional transbronchial needle aspiration vs endobronchial ultrasound guided transbronchial needle aspiration with and without rapid onsite evaluation in a single center. Chest. 2009;136:112S.

28. Mitsudomi T, Morita S, Yatabe Y, et al. Gefitinib versus cisplatin plus docetaxel in patients with nonsmall-cell lung cancer harbouring mutations of the epidermal growth factor receptor (WJTOG3405): an open label, randomised phase 3 trial. Lancet Oncol. 2010;11:121-128.

29. Mok TS, Wu YL, Thongprasert S, et al. Gefitinib or carboplatinpaclitaxel in pulmonary adenocarcinoma. $N$ Engl J Med. 2009;361: 947-957.

30. Nakajima T, Yasufuku K. How I do it—optimal methodology for multidirectional analysis of endobronchial ultrasound-guided transbronchial needle aspiration samples. J Thorac Oncol. 2011;6:203-206. 
31. Casal RF, Staerkel GA, Ost D, et al. Randomized clinical trial of endobronchial ultrasound new biopsy with and without aspiration. Chest. 2012;142:568-573.

32. 22-gauge ProCore Needle v. Standard 22-gauge (P00030500). Available from: https://clinicaltrials.gov/ct2/show/NCT02154698. Accessed December 18, 2015.

33. Desai NR, Greenhill SR, Vance M, Kovitz KL. Endobronchial ultrasound guided transbronchial core needle biopsy using a novel needle. AJRCCM. 185;2012:A1105.

34. Rintoul RC, Tournoy KG, El Daly H, et al. EBUSTBNA for the clarification of PET positive intrathoracic lymph nodes - an international multi-centre experience. J Thorac Oncol. 2009;4:44-48.

35. Zambon M, Nagarathiam R, Russel D, Giampoli E, Nead M. ProCore needles vs standard 22 gauge needle in EBUS guided biopsies. Chest. 2015;148(4):791A.

36. Izuma T, Sasada S, Watanabe J, Chavez C, Matsumoto Y, Tsuchida T. Comparison of 22-gauge aspiration needles for histologic sampling during endobronchial ultrasound guided transbronchial needle aspiration. ERJ. 2014;44(S58).

37. Witt BL, Adler DG, Hilden K, et al. A comparative needle study: EUSFNA procedures using the HD ProCore and EchoTip 22 gauge needle types. Diagn Cytopathol. 2013;41:1069-1074.

38. Lee HS, Lee GK, Lee HS, et al. Real-time endobronchial ultrasoundguided transbronchial needle aspiration in mediastinal staging of nonsmall cell lung cancer: how many aspirations per target lymph node station? Chest. 2008;134:368-374.
39. Trisolini R, Cancellieri A, Tinelli C, et al. Rapid on-site evaluation of transbronchial aspirates in the diagnosis of hilar and mediastinal adenopathy: a randomized trial. Chest. 2011;139:395-401.

40. Nakajima T, Yasufuku K, Takahashi R, et al. Comparison of 21-gauge and 22-gauge aspiration needle during endobronchial ultrasound-guided transbronchial needle aspiration. Respirology. 2011;16:90-94.

41. Prakash UB. A better bronchoscopic technique to obtain diagnostic tissue from the mediastinal lymph nodes. J Bronchol. 2005;12:1-2.

42. Oki M, Saka H, Sako C. Bronchoscopic miniforceps biopsy for mediastinal nodes. J Bronchol. 2004;11:150-153.

43. Chrissian A, Misselhorn D, Chen A. Endobronchial-ultrasound guided miniforceps biopsy of mediastinal and hilar lesions. Ann Thorac Surg. 2011;92:284-288.

44. Herth FJF, Schuler H, Gompelmann D, et al. Endobronchial ultrasoundguided lymph node biopsy with transbronchial needle forceps: a pilot study. Eur Respir J. 2012;39:373-377.

45. Darwiche K, Freitag L, Nair A, et al. Evaluation of a novel endobronchial ultrasound-guided lymph node forceps in enlarged mediastinal lymph nodes. Respiration. 2013;86:229-236.
Medical Devices: Evidence and Research

\section{Publish your work in this journal}

Medical Devices: Evidence and Research is an international, peerreviewed, open access journal that focuses on the evidence, technology, research, and expert opinion supporting the use and application of medical devices in the diagnosis, treatment and management of clinical conditions and physiological processes. The identification of novel

\section{Dovepress}

devices and optimal use of existing devices which will lead to improved clinical outcomes and more effective patient management and safety is a key feature. The manuscript management system is completely online and includes a quick and fair peer-review system. Visit http://www. dovepress.com/testimonials.php to read real quotes from authors. 